\title{
Abnormal metabolism of secondary bile acids in patients with cirrhosis ${ }^{1}$
}

\author{
Z. R. VLAHCEVIC, I. BUHAC, C. C. BELL, Jr, AND LEON SWELL \\ From the Veterans Administration Hospital and the Medical College of Virginia, USA
}

SUMMARY The composition of bile acids in human bile was determined in bile-rich duodenal fluid on four consecutive days in a group of seven patients with cirrhosis and $\overrightarrow{0}$ eight control patients with no liver disease. There was a marked reduction of secondary biliary bile acids in cirrhotic patients. Possible mechanisms for these changes are discussed.

The main bile acids in human bile are cholic, chenodeoxycholic, and deoxycholic with a mean ratio of $1 \cdot 1: 1 \cdot 0: 0 \cdot 6$ (Sjövall, 1960). Cholic and chenodeoxycholic acid are primary bile acids synthesized from cholesterol in the liver (Bergström, Danielsson, and Samuelsson, 1960). These bile acids are not interconvertible. The secondary bile acids, deoxycholic and lithocholic, are products formed by the action of intestinal microbial enzymes. The removal of the hydroxyl group at C-7 position of cholic and chenodeoxycholic acid is effected mainly by anaerobic intestinal microorganisms resulting in the formation of deoxycholic and lithocholic acids respectively (Norman and Bergman, 1960; Bergström et al, 1960; Hill and Drasar, 1968). Deoxycholic acid occurs in varying amounts in the bile of several species and is the major bile acid found in human faeces. Lithocholic acid is poorly absorbed by the colon and is therefore present in small amounts in human bile. Following the dehydroxylation of the primary bile acids, the secondary bile acids enter the enterohepatic cycle and are incorporated into the total bile salt pool. In man and the rabbit, the secondary bile acids are not rehydroxylated in the liver (Ekdahl and Sjövall, 1955; Lindstedt and Sjövall, 1957).

In the light of our present knowledge, the presence of the secondary bile acids in human bile is a reflection of normal intestinal flora and

${ }^{1}$ Requests for reprints should be sent to $\operatorname{Dr} Z$. R. Vlahcevic, Gastroenterology Section, McGuire Veterans Administration Hospital, Richmond, Virginia 23219, USA. undisturbed enterohepatic circulation. The ab- $\stackrel{\mathbb{Q}}{2}$ sence of secondary bile acids in bile can occur $\overrightarrow{\overrightarrow{0}}$ in subjects with a complete biliary fistula (Ekdahl and Sjövall, 1957), germ-free animals (Gustafsson, Bergström, Lindstedt, and Norman, 1957), or subjects on oral antibiotics (Hamilton, 1963).

In the course of studies on bile acid metabolism: in patients with cirrhosis it was noted that the secondary bile acids in bile were almost completely absent. In view of the paucity of the information on the metabolism of bile acids ino liver disease, it was of interest to examine the biliary bile acid composition in a group of cirrhotic patients compared with patients without $\frac{\bar{N}}{\mathrm{~N}}$ liver disease. The data indicate that patients. with cirrhosis have an abnormality in the N metabolism of secondary bile acids.

\section{Methods and Materials}

Two groups of patients were used in these $\frac{{ }^{\circ}}{\overrightarrow{0}}$ studies. The control group of eight patients had

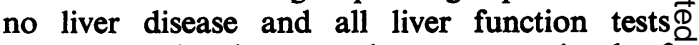
were normal. The second group consisted of seven patients with varying degrees of cirrhosis of the alcoholic. Cirrhosis was classified as advanced, moderate, and mild on the basis of histological appearance of the liver biopsy and을 liver function tests, and clinical signs such as the presence or absence of ascites, portal hypertension, and varices. During the study the patients did not receive antibiotic therapy 


\begin{tabular}{|c|c|c|c|c|}
\hline \multirow[t]{3}{*}{ Patient } & \multicolumn{4}{|c|}{ Bile Acid Composition as percentages of Total Bile Acids ${ }^{1}$} \\
\hline & \multicolumn{2}{|c|}{ Primary Bile Acids } & \multicolumn{2}{|c|}{ Secondary Bile Acids } \\
\hline & Cholic & Chenodeoxycholic & Deoxycholic & Lithocholic \\
\hline \multicolumn{5}{|c|}{ Patients without liver disease } \\
\hline $\begin{array}{l}\text { W.S. } \\
\text { H.F. } \\
\text { G.H. } \\
\text { W.B. } \\
\text { E.B. } \\
\text { C.H. } \\
\text { C.P. } \\
\text { W.A. }\end{array}$ & $\begin{array}{l}35 \cdot 4 \\
66 \cdot 7 \\
42 \cdot 4 \\
56 \cdot 1 \\
45 \cdot 3 \\
67 \cdot 2 \\
47 \cdot 4 \\
43 \cdot 0\end{array}$ & $\begin{array}{l}54 \cdot 4 \\
30 \cdot 5 \\
37 \cdot 3 \\
34 \cdot 1 \\
36 \cdot 3 \\
24 \cdot 5 \\
37 \cdot 9 \\
32 \cdot 6\end{array}$ & $\begin{array}{r}8 \cdot 8 \\
2 \cdot 3 \\
17 \cdot 8 \\
6 \cdot 0 \\
12 \cdot 0 \\
8 \cdot 0 \\
11 \cdot 9 \\
22 \cdot 1\end{array}$ & $\begin{array}{l}1 \cdot 4 \\
0 \cdot 5 \\
2 \cdot 4 \\
3 \cdot 8 \\
6 \cdot 4 \\
0 \cdot 3 \\
2 \cdot 8 \\
2 \cdot 2\end{array}$ \\
\hline Mean & $50 \cdot 4 \pm 12 \cdot 0$ & $36 \cdot 0 \pm 8 \cdot 6$ & $11 \cdot 1 \pm 6 \cdot 4$ & $2 \cdot 5 \pm 2.0$ \\
\hline \multicolumn{5}{|c|}{ Cirrhotic patients } \\
\hline $\begin{array}{l}\text { L.W. } \\
\text { E.D. } \\
\text { A.G. } \\
\text { E.L. } \\
\text { N.S. } \\
\text { M.Y. } \\
\text { E.H. }\end{array}$ & $\begin{array}{l}66 \cdot 9 \\
31 \cdot 9 \\
43 \cdot 3 \\
46 \cdot 4 \\
60 \cdot 3 \\
50 \cdot 5 \\
63 \cdot 4\end{array}$ & $\begin{array}{l}32 \cdot 3 \\
67.4 \\
55 \cdot 8 \\
52.4 \\
39.1 \\
44.6 \\
29.0\end{array}$ & $\begin{array}{l}0.5 \\
0.3 \\
0.6 \\
0.8 \\
0.3 \\
3.8 \\
7 \cdot 2\end{array}$ & $\begin{array}{l}0 \cdot 3 \\
0 \cdot 4 \\
0 \cdot 3 \\
0 \cdot 4 \\
0 \cdot 3 \\
1 \cdot 1 \\
0 \cdot 4\end{array}$ \\
\hline $\begin{array}{l}\text { Mean } \\
\mathbf{P}\end{array}$ & $\begin{array}{l}51 \cdot 8 \pm 12 \cdot 4 \\
\text { Not significant }\end{array}$ & $\begin{array}{l}45 \cdot 8 \pm 13 \cdot 7 \\
\text { Not significant }\end{array}$ & $\begin{array}{l}1.9 \pm 2.6 \\
<0.005\end{array}$ & $\begin{array}{l}0.5 \pm 0.3 \\
<0.025\end{array}$ \\
\hline
\end{tabular}

Table Composition of biliary bile acids in normal and cirrhotic patients

${ }^{1}$ Values in each patient represent the average of four bile samples obtained on four consecutive days.

nor did they have diarrhoea. All patients were placed in a metabolic ward and maintained on a standard 2,500 calorie hospital diet consisting of $92 \mathrm{~g}$ of protein, $232 \mathrm{~g}$ of carbohydrate, and $80 \mathrm{~g}$ of fat for four days before the start of the study. Bile-rich duodenal fluid was obtained using a Dreiling double-lumen tube. The bile was obtained after the gall bladder, stimulated by either a fatty meal or cholecystokinin, had been emptied. Bile specimens were obtained on four consecutive days. Sjövall (1960) showed that bile acid composition of bile-rich duodenal fluid is representative of gallbladder bile; the ratio of cholic:chenodeoxycholic acid in subjects without liver disease was $1 \cdot 0: 1 \cdot 0: 0 \cdot 5$ for gallbladder bile and $1 \cdot 1: 1 \cdot 0: 0 \cdot 6$ for bile-enriched duodenal contents.

Bile salts were extracted with 20 volumes 2:1 chloroform methanol and partitioned with 0.2 volumes of water (Folch, Lees, and SloaneStanley, 1957). Bile acid analysis was performed on the methanol-water phase (Swell, 1968). An aliquot of the water-methanol phase was autoclaved with $2.5 \mathrm{~N} \mathrm{KOH}$ for three hours at $15 \mathrm{lb}$ pressure and acidified to $p \mathrm{H} 1.0$ with $\mathrm{HCl}$. The bile acids were extracted with diethyl ether. The methyl esters of the bile acids were prepared with diazomethane. The TMS derivatives of the bile acid methyl esters were prepared and subjected to gas liquid chromatography using cholestane as the internal standard as described earlier (Swell, 1968). Purified bile acid standards were obtained from Applied Science Laboratories, USA.

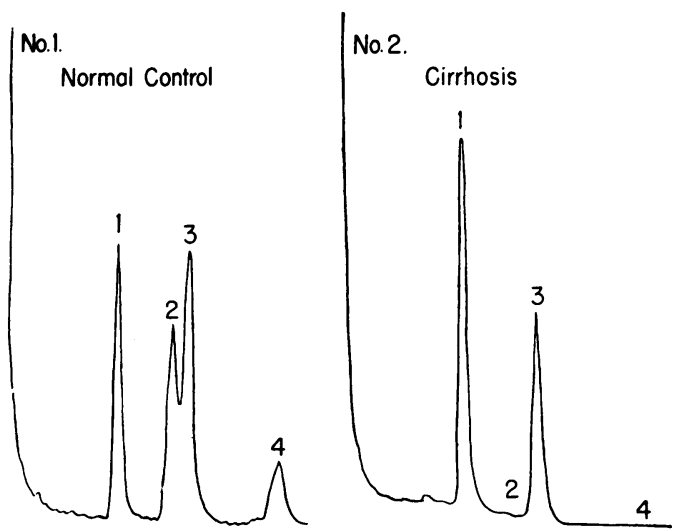

Fig. Gas liquid chromatogram of biliary bile acids of a normal control (no. l) and a cirrhotic patient (no. 2). The peaks represent the following bile acids: 1 cholic, 2 deoxycholic, 3 chenodeoxycholic, and 4 lithocholic.

\section{Results}

The table shows the distribution of bile acids in the control patients without liver disease and patients with cirrhosis expressed as a percentage of the total bile acids. Each value represents the mean value of four consecutive determinations in the same patient. The day-to-day variation in bile acid composition in a given individual was very small. Patients with cirrhosis had a markedly lower percentage of biliary deoxycholic and lithocholic acids than patients without liver disease. This difference was highly significant for deoxycholic acid $(P<0.005)$ and lithocholic acid $(P<0.025)$. Five of the seven cirrhotic patients had only traces (less than $1 \%$ ) of deoxycholic acid. There was no correlation between the severity of liver disease as judged by clinical criteria, liver function tests, and the percentage of deoxycholic acid. The mean percentage of cholic acid in both groups was essentially the same $(51.8$ versus 50.4$)$. The cirrhotic patients had a higher percentage of chenodeoxycholic acid than the control group ( 45.8 versus 36.0$)$ but this difference was not statistically significant.

The figure shows several representative examples of bile acid distribution patterns obtained by gas liquid chromatography on a normal and a cirrhotic patient. The cirrhotic patient shows a complete absence of secondary bile acids.

\section{Discussion}

Sjövall (1960), using paper chromatographic techniques, studied the composition and ratio 
of conjugated bile acids in healthy subjects and patients with various hepato-biliary diseases. He observed that patients with liver disease and biliary obstruction had a decreased ratio of glycine- to taurine-conjugated bile acids, and also a reduction in the level of biliary deoxycholic acid. The results of the present study have provided additional evidence that in patients with cirrhosis the metabolism of secondary bile acids is markedly impaired. Recent preliminary findings have shown that patients with cirrhosis also have a reduction in primary bile acid synthesis and total bile salt pool. Thus it appears that patients with cirrhosis have a defect in the metabolism of both primary and secondary bile acids which could result in a decreased total amount of bile salts presented to the small intestine. This could lead to inadequate micellar solubilization of intestinal lipid and subsequent steatorrhea (Badley, Murphy, Bouchier, and Sherlock, 1969). Several possibilities can be offered to explain the marked reduction or absence of biliary deoxycholic acid in patients with cirrhosis. A change in the composition of the intestinal microbial flora could effect the conversion of cholic and chenodeoxycholic to deoxycholic and lithocholic acids. The absorption of secondary bile acids from the colon may be greatly reduced in these patients, and finally the diseased liver may be unable to conjugate and/or excrete circulating deoxycholic and lithocholic acids. Further studies are being carried out to provide information on these points.
References

Badley, B. W. D., Murphy, G. M., Bouchier, I. A. D., and $\Omega$ Sherlock, S. (1969). The role of bile salts in the steatorrhoea of chronic liver disease. (abstr.) Gastroenterology, 56, 1136.

Bergström, S., Danielson, H., and Samuelsson, B. (1960). Formation and metabolism of bile acids. In Lipid Metabolism, edited by K. Bloch, pp. 291-331. Wiley, New York.

Ekdahl, P. H., and Sjövall, J. (1955). Metabolism of desoxycholic $\overline{\bar{G}}$ acid in the rabbit. Bile acids and steroids 28. Acta physiol. scand., 34, 287-294.

Ekdahl, P. H., and Sjövall, J. (1958). On the conjugation and formation of bile acids in the human liver. I. On the $\mathcal{C}$ excretion of bile acids by patients with postoperative choledochostomy drainage. Bile acids and steroids 61. Acta chir. scand., 114, 439-452.

Folch, J., Lees, M., and Sloane Stanley, G. H. (1957). A simple $\vec{\omega}$ method for the isolation and purification of total lipids $D$ from animal tissues. J. biol. Chem., 226, 497-509.

Gustafsson, B. E., Bergström, S., Lindstedt, S., and Norman, A. (1957). Turnover and nature of fecal bile acids in germfree and infected rats fed cholic acid-24-14C. Bile acids and $\vec{r}$

Hamilton, J. G. (1963). The effect of oral neomycin on the $A$ conversion of cholic acid to deoxycholic in man. Arch. $N$ Biochem. Biophys., 101, 7-13.

Hill, M. J., and Drasar, B. S. (1968). Degradation of bile salts by human intestinal bacteria. Gut, 9,22-27.

Lindstedt, S., and Sjövall, J. (1957). On the formation of deoxy- $z$ cholic acid from cholic acid in the rabbit. Bile acids and 0 steroids 48. Acta chem. scand., 11, 421-426.

Norman, A., and Bergman, S. (1960). The action of intestinal $\overrightarrow{0}$ micro-organisms on bile acids. Bile acids and steroids 101. Acta chem. scand., 14, 1781-1789.

Sjövall, J. (1960). Bile acids in man under normal and pathological conditions. Bile acids and steroids 73. Clin. chim. Acta , 5, 33-41.

Swell, L. (1968). Separation, identification, and estimation of radioactive and nonradioactive lipids. In Theory and Application of Gas Chromatography, edited by H. S. Kroman and S. R. Bender, pp. 97-109. Grune \& Stratton, New York. 